\title{
QUASILINEARITY OF SOME COMPOSITE FUNCTIONALS WITH APPLICATIONS
}

\author{
S. S. DRAGOMIR
}

(Received 8 March 2010)

\begin{abstract}
The quasilinearity of certain composite functionals defined on convex cones in linear spaces is investigated. Applications in refining the Jensen, Hölder, Minkowski and Schwarz inequalities are given.

2000 Mathematics subject classification: primary 26D15; secondary 26D10.

Keywords and phrases: additive, superadditive and subadditive functionals, convex functions, Jensen's inequality, Hölder's inequality, Minkowski's inequality, Schwarz's inequality.
\end{abstract}

\section{Introduction}

Let $X$ be a linear space. A subset $C \subseteq X$ is called a convex cone in $X$ provided the following conditions hold:

(i) $\quad x, y \in C$ imply $x+y \in C$;

(ii) $x \in C, \alpha \geq 0$ imply $\alpha x \in C$.

A functional $h: C \rightarrow \mathbb{R}$ is said to be superadditive (subadditive) on $C$ if:

(iii) $h(x+y) \geq(\leq) h(x)+h(y)$ for any $x, y \in C$,

and nonnegative (strictly positive) on $C$ if it satisfies:

(iv) $h(x) \geq(>) 0$ for each $x \in C$.

The functional $h$ is $s$-positive homogeneous on $C$, for a given $s>0$, if:

(v) $h(\alpha x)=\alpha^{s} h(x)$ for any $\alpha \geq 0$ and $x \in C$.

In [3], the following result has been obtained.

THEOREM 1.1. Let $x, y \in C$ and let $h: C \rightarrow \mathbb{R}$ be a nonnegative, superadditive and s-positive homogeneous functional on $C$. If $M \geq m \geq 0$ are such that $x-m y$ and $M y-x \in C$, then

$$
M^{s} h(y) \geq h(x) \geq m^{s} h(y) .
$$

Now, consider $v: C \rightarrow \mathbb{R}$ to be an additive and strictly positive functional on $C$ which is also positive homogeneous on $C$. The following result concerning other bounds for a composite functional may be stated as well [3].

(C) 2010 Australian Mathematical Publishing Association Inc. 0004-9727/2010 \$16.00 
THEOREM 1.2. Let $x, y \in C$, let $h$ be strictly positive, superadditive and positive homogeneous on $C$ and let $v$ be an additive, strictly positive and positive homogeneous functional on $C$. If $M \geq m>0$ are such that $x-m y$ and $M y-x \in C$, then

$$
\left[\frac{h(y)}{v(y)}\right]^{M v(y)} \geq\left[\frac{h(x)}{v(x)}\right]^{v(x)} \geq\left[\frac{h(y)}{v(y)}\right]^{m v(y)} .
$$

As shown in [3], the above results can be applied to obtain refinements of the Jensen, Hölder, Minkowski and Schwarz inequalities for weights satisfying certain conditions.

The main aim of the present paper is to study the quasilinearity properties of other composite functionals and to apply the obtained results to improving some classical inequalities as those mentioned above.

\section{General results}

The following result provides the quasilinearity property of a composite functional when one of the components is additive while the other is either superadditive or subadditive.

THEOREM 2.1. Let $C$ be a convex cone in the linear space $X$ and let $v: C \rightarrow(0, \infty)$ be an additive functional on $C$. If $h: C \rightarrow[0, \infty)$ is a superadditive (subadditive) functional on $C$ and $p \geq 1(0<p<1)$, then the functional

$$
\Psi_{p}: C \rightarrow[0, \infty), \quad \Psi_{p}(x)=v^{1-1 / p}(x) h(x)
$$

is superadditive (subadditive) on $C$.

PROOF. First of all, we observe that the elementary inequality

$$
(\alpha+\beta)^{p} \geq(\leq) \alpha^{p}+\beta^{p}
$$

holds for any $\alpha, \beta \geq 0$ and $p \geq 1(0<p<1)$.

Indeed, if we consider the function

$$
f_{p}:[0, \infty) \rightarrow \mathbb{R}, \quad f_{p}(t)=(t+1)^{p}-t^{p}
$$

we have $f_{p}^{\prime}(t)=p\left[(t+1)^{p-1}-t^{p-1}\right]$. Observe that for $p>1$ and $t>0$ we have that $f_{p}^{\prime}(t)>0$, which shows that $f_{p}$ is strictly increasing on the interval $[0, \infty)$. Now for $t=\alpha / \beta$ (where $\beta>0$ and $\alpha \geq 0$ ) we have $f_{p}(t)>f_{p}(0)$ and consequently $((\alpha / \beta)+1)^{p}-(\alpha / \beta)^{p}>1$, which is the desired inequality (2.2).

For $p \in(0,1)$ we have that $f_{p}$ is strictly decreasing on $[0, \infty)$ which proves the second case in (2.2).

Now, if $h$ is superadditive (subadditive) and $p \geq 1(0<p<1)$, then we have by (2.2) that

$$
h^{p}(x+y) \geq(\leq)[h(x)+h(y)]^{p} \geq(\leq) h^{p}(x)+h^{p}(y)
$$

for all $x, y \in C$. 
Utilizing (2.3) and the additivity property of $v$, we have for any $x, y \in C$ that

$$
\begin{aligned}
\frac{h^{p}(x+y)}{v(x+y)} & \geq(\leq) \frac{h^{p}(x)+h^{p}(y)}{v(x)+v(y)} \\
& =\frac{v(x) \cdot \frac{h^{p}(x)}{v(x)}+v(y) \cdot \frac{h^{p}(y)}{v(y)}}{v(x)+v(y)} \\
& =\frac{v(x) \cdot\left[\frac{h(x)}{v^{1 / p}(x)}\right]^{p}+v(y) \cdot\left[\frac{h(y)}{v^{1 / p}(y)}\right]^{p}}{v(x)+v(y)}=: I .
\end{aligned}
$$

Since for $p \geq 1(0<p<1)$ the power function $g(t)=t^{p}$ is convex (concave), then

$$
\begin{aligned}
I & \geq(\leq)\left[\frac{v(x) \cdot \frac{h(x)}{v^{1 / p}(x)}+v(y) \cdot \frac{h(y)}{v^{1 / p}(y)}}{v(x)+v(y)}\right]^{p} \\
& =\left[\frac{h(x) v^{1-1 / p}(x)+h(y) v^{1-1 / p}(y)}{v(x+y)}\right]^{p}
\end{aligned}
$$

for any $x, y \in C$.

By combining (2.4) with (2.5) we get

$$
\frac{h^{p}(x+y)}{v(x+y)} \geq(\leq)\left[\frac{h(x) v^{1-1 / p}(x)+h(y) v^{1-1 / p}(y)}{v(x+y)}\right]^{p}
$$

which is equivalent to

$$
\frac{h(x+y)}{v^{1 / p}(x+y)} \geq(\leq) \frac{h(x) v^{1-1 / p}(x)+h(y) v^{1-1 / p}(y)}{v(x+y)}
$$

that is, by multiplying by $v(x+y)$,

$$
\Psi_{p}(x+y) \geq(\leq) \Psi_{p}(x)+\Psi_{p}(y)
$$

for any $x, y \in C$ and the proof is complete.

Corollary 2.2. Assume that $X, C$ and $v$ are as in Theorem 2.1. If $h: C \rightarrow[0, \infty)$ is a superadditive (subadditive) functional on $C$ and $p, q \geq 1(0<p, q<1)$, then the two-parameter functional

$$
\Psi_{p, q}: C \rightarrow[0, \infty), \quad \Psi_{p, q}(x)=v^{q(1-(1 / p))}(x) h^{q}(x)
$$

is superadditive (subadditive) on $C$.

Proof. Observe that $\Psi_{p, q}(x)=\left[\Psi_{p}(x)\right]^{q}$ for $x \in C$. Therefore, by Theorem 2.1 and the inequality (2.2), for $q \geq 1(0<q<1)$ we have

$$
\begin{aligned}
\Psi_{p, q}(x+y) & =\left[\Psi_{p}(x+y)\right]^{q} \geq(\leq)\left[\Psi_{p}(x)+\Psi_{p}(y)\right]^{q} \\
& \geq(\leq)\left[\Psi_{p}(x)\right]^{q}+\left[\Psi_{p}(y)\right]^{q}=\Psi_{p, q}(x)+\Psi_{p, q}(y)
\end{aligned}
$$

for any $x, y \in C$ and the statement is proved. 
REMARK 2.3. If we consider the functional $\psi_{p}(x):=v^{p-1}(x) h^{p}(x)$, then for $p \geq 1$ $(0<p<1)$ and $h: C \rightarrow[0, \infty)$ a superadditive (subadditive) functional on $C$, the functional $\psi_{p}$ is also superadditive (subadditive) on $C$.

The following result provides upper and lower bounds for a value of the functional $\Psi_{p, q}$ in the case when the composite functionals are homogeneous.

COROLlary 2.4. Let $x, y \in C, h: C \rightarrow \mathbb{R}$ be a nonnegative, superadditive and s-positive homogeneous functional on $C$ and $v$ an additive, strictly positive and positive homogeneous functional on $C$. If $p, q \geq 1$ and $M \geq m \geq 0$ are such that $x-m y$ and $M y-x \in C$, then

$$
M^{s q+q(1-1 / p)} \Psi_{p, q}(y) \geq \Psi_{p, q}(x) \geq m^{s q+q(1-1 / p)} \Psi_{p, q}(y) .
$$

In particular,

$$
M^{(s+1) p-1} \psi_{p}(y) \geq \psi_{p}(x) \geq m^{(s+1) p-1} \psi_{p}(y)
$$

and

$$
M^{s+1-1 / p} \Psi_{p}(y) \geq \Psi_{p}(x) \geq m^{s+1-1 / p} \Psi_{p}(y),
$$

where $\psi_{p}$ and $\Psi_{p}$ are defined as above.

PROOF. The proof follows from Theorem 1.1 observing, from Corollary 2.2, that the functional $\Psi_{p, q}$ is superadditive and $[s q+q(1-(1 / p))]$-positive homogeneous, where $s>0$ and $p, q \geq 1$.

The following result also holds.

THEOREM 2.5. Let $C$ be a convex cone in the linear space $X$ and $v: C \rightarrow(0, \infty)$ an additive functional on $C$. If $h: C \rightarrow(0, \infty)$ is a superadditive functional on $C$ and $0<p<1$, then the functional

$$
\Phi_{p}: C \rightarrow(0, \infty), \quad \Phi_{p}(x)=\frac{1}{v^{(1 / p)-1}(x) h(x)}
$$

is subadditive on $C$.

PROOF. Let $s:=-p \in[-1,0)$. For $s<0$ we have the inequality

$$
(\alpha+\beta)^{s} \leq \alpha^{s}+\beta^{s}
$$

for any $\alpha, \beta>0$.

Indeed, by the convexity of the function $f_{s}(t)=t^{s}$ on $(0, \infty)$ with $s<0$, we have that

$$
(\alpha+\beta)^{s} \leq 2^{s-1}\left(\alpha^{s}+\beta^{s}\right)
$$

for any $\alpha, \beta>0$ and since, obviously, $2^{s-1}\left(\alpha^{s}+\beta^{s}\right) \leq \alpha^{s}+\beta^{s}$, then (2.11) holds.

Taking into account the fact that $h$ is superadditive, then by (2.11)

$$
h^{s}(x+y) \leq[h(x)+h(y)]^{s} \leq h^{s}(x)+h^{s}(y)
$$

for any $x, y \in C$. 
Since $v$ is additive, then by (2.11) we have that

$$
\begin{aligned}
\frac{h^{s}(x+y)}{v(x+y)} & \leq \frac{h^{s}(x)+h^{s}(y)}{v(x)+v(y)} \\
& =\frac{v(x) \cdot\left[\frac{h(x)}{v^{1 / s}(x)}\right]^{s}+v(y) \cdot\left[\frac{h(y)}{v^{1 / s}(y)}\right]^{s}}{v(x)+v(y)} \\
& =\frac{v(x) \cdot\left[\frac{v^{1 / s}(x)}{h(x)}\right]^{-s}+v(y) \cdot\left[\frac{v^{1 / s}(y)}{h(y)}\right]^{-s}}{v(x)+v(y)}=: J .
\end{aligned}
$$

By the concavity of the function $g(t)=t^{-s}$ with $s \in[-1,0)$ we also have

$$
J \leq\left[\frac{v(x) \cdot \frac{v^{1 / s}(x)}{h(x)}+v(y) \cdot \frac{v^{1 / s}(y)}{h(y)}}{v(x)+v(y)}\right]^{-s} .
$$

Making use of (2.13) and (2.14) we get

$$
\frac{h^{s}(x+y)}{v(x+y)} \leq\left[\frac{v(x) \cdot \frac{v^{1 / s}(x)}{h(x)}+v(y) \cdot \frac{v^{1 / s}(y)}{h(y)}}{v(x)+v(y)}\right]^{-s}
$$

for any $x, y \in C$, which is equivalent to

$$
\frac{h^{-1}(x+y)}{v^{-1 / s}(x+y)} \leq \frac{\frac{v^{1+1 / s}(x)}{h(x)}+\frac{v^{1+1 / s}(y)}{h(y)}}{v(x)+v(y)}
$$

and, since $v(x+y)=v(x)+v(y)$, to

$$
\frac{v^{1+1 / s}(x+y)}{h(x+y)} \leq \frac{v^{1+1 / s}(x)}{h(x)}+\frac{v^{1+1 / s}(y)}{h(y)}
$$

for any $x, y \in C$.

This completes the proof.

The following result may also be stated.

Corollary 2.6. Assume that $X, C$ and $v$ are as in Theorem 2.5. If $h: C \rightarrow(0, \infty)$ is a superadditive functional on $C$ and $0<p, q<1$, then the functional

$$
\Phi_{p, q}: C \rightarrow(0, \infty), \quad \Phi_{p, q}(x)=\frac{1}{v^{q((1 / p)-1)}(x) h^{q}(x)}
$$

is subadditive on $C$.

Proof. Observe that $\Phi_{p, q}(x)=\left[\Phi_{p}(x)\right]^{q}$ for $x \in C$. Therefore, by Theorem 2.5 and the inequality (2.2) for $0<q<1$, we have that

$$
\begin{aligned}
\Phi_{p, q}(x+y) & =\left[\Phi_{p}(x+y)\right]^{q} \leq\left[\Phi_{p}(x)+\Phi_{p}(y)\right]^{q} \\
& \leq\left[\Phi_{p}(x)\right]^{q}+\left[\Phi_{p}(y)\right]^{q}=\Phi_{p, q}(x)+\Phi_{p, q}(y)
\end{aligned}
$$

for any $x, y \in C$ and the statement is proved. 
REMARK 2.7. If we consider the functional

$$
\varphi_{p}(x):=\frac{1}{v^{1-p}(x) h^{p}(x)}
$$

then, for $0<p<1$ and $h: C \rightarrow(0, \infty)$ a superadditive functional on $C$, the functional $\varphi_{p}$ is subadditive on $C$.

\section{Applications for Jensen's inequality}

Let $C$ be a convex subset of the real linear space $X$ and let $f: C \rightarrow \mathbb{R}$ be a convex mapping. Here we consider the following well-known form of Jensen's discrete inequality:

$$
f\left(\frac{1}{P_{I}} \sum_{i \in I} p_{i} x_{i}\right) \leq \frac{1}{P_{I}} \sum_{i \in I} p_{i} f\left(x_{i}\right)
$$

where $I$ denotes a finite subset of the set $\mathbb{N}$ of natural numbers, $x_{i} \in C, p_{i} \geq 0$ for $i \in I$ and $P_{I}:=\sum_{i \in I} p_{i}>0$.

Let us fix $I \in \mathcal{P}_{f}(\mathbb{N})$ (the class of finite parts of $\mathbb{N}$ ) to be a set consisting of two or more indices and $x_{i} \in C(i \in I)$. Now consider the functional $J: S_{+}(I) \rightarrow \mathbb{R}$ given by

$$
J_{I}(\mathbf{p}):=\sum_{i \in I} p_{i} f\left(x_{i}\right)-P_{I} f\left(\frac{1}{P_{I}} \sum_{i \in I} p_{i} x_{i}\right) \geq 0,
$$

where

$$
S_{+}(I):=\left\{\mathbf{p}=\left(p_{i}\right)_{i \in I} \mid p_{i} \geq 0, i \in I \text { and } P_{I}>0\right\}
$$

and $f$ is convex on $C$.

We observe that $S_{+}(I)$ is a convex cone and the functional $J_{I}$ is nonnegative and positive homogeneous on $S_{+}(I)$.

LEMMA 3.1 [5]. The functional $J_{I}(\cdot)$ is a superadditive functional on $S_{+}(I)$.

Define the functional

$$
J_{p, q, I}(\mathbf{p}):=\left[P_{I}^{(p-1) / p} \sum_{i \in I} p_{i} f\left(x_{i}\right)-P_{I}^{(2 p-1) / p} f\left(\frac{1}{P_{I}} \sum_{i \in I} p_{i} x_{i}\right)\right]^{q}
$$

for $p>1$ and $q \geq 1$.

The following proposition can be stated.

PROPOSITION 3.2. The functional $J_{p, q, I}(\cdot)$ is superadditive on $S_{+}(I)$ for any $p>1$ and $q \geq 1$.

Proof. Define $v(\mathbf{p})=P_{I}$ and $h(\mathbf{p})=J_{I}(\mathbf{p})$. Then, for $p>1$ and $q \geq 1$,

$$
\Psi_{p, q}(\mathbf{p})=v^{q(1-(1 / p))}(\mathbf{p}) h^{q}(\mathbf{p})=P_{I}^{q(1-(1 / p))} J_{I}^{q}(\mathbf{p})=J_{p, q, I}(\mathbf{p})
$$

for any $\mathbf{p} \in S_{+}(I)$.

Since $v(\cdot)$ is additive and $J_{I}(\cdot)$ is superadditive on $S_{+}(I)$, on applying Corollary 2.2 we conclude that $J_{p, q, I}(\cdot)$ is also superadditive on $S_{+}(I)$. 
REMARK 3.3. We observe that, in particular, the functionals

$$
J_{p, I}(\mathbf{p}):=P_{I}^{(p-1) / p} \sum_{i \in I} p_{i} f\left(x_{i}\right)-P_{I}^{(2 p-1) / p} f\left(\frac{1}{P_{I}} \sum_{i \in I} p_{i} x_{i}\right)
$$

and

$$
\tilde{J}_{p, I}(\mathbf{p}):=\left[P_{I}^{(p-1) / p} \sum_{i \in I} p_{i} f\left(x_{i}\right)-P_{I}^{(2 p-1) / p} f\left(\frac{1}{P_{I}} \sum_{i \in I} p_{i} x_{i}\right)\right]^{p}
$$

are superadditive on $S_{+}(I)$ for any $p>1$.

The following result provides a refinement and a reverse for Jensen's inequality when bounds for the weights are known.

Proposition 3.4. If $\mathbf{p}, \mathbf{q} \in S_{+}(I)$ and $M \geq m \geq 0$ are such that $M \mathbf{p} \geq \mathbf{q} \geq m \mathbf{p}$, that is, $M p_{i} \geq q_{i} \geq m p_{i}$ for each $i \in I$, then

$$
\begin{aligned}
& M^{(2 p-1) / p}\left(\frac{P_{I}}{Q_{I}}\right)^{(p-1) / p}\left[\sum_{i \in I} p_{i} f\left(x_{i}\right)-P_{I} f\left(\frac{1}{P_{I}} \sum_{i \in I} p_{i} x_{i}\right)\right] \\
& \geq \sum_{i \in I} q_{i} f\left(x_{i}\right)-Q_{I} f\left(\frac{1}{Q_{I}} \sum_{i \in I} q_{i} x_{i}\right) \\
& \geq m^{(2 p-1) / p}\left(\frac{P_{I}}{Q_{I}}\right)^{(p-1) / p}\left[\sum_{i \in I} p_{i} f\left(x_{i}\right)-P_{I} f\left(\frac{1}{P_{I}} \sum_{i \in I} p_{i} x_{i}\right)\right],
\end{aligned}
$$

for any $p>1$.

PROOF. Applying Corollary 2.4 for the functional $\Psi_{p, q}(p)=J_{p, q, I}(\mathbf{p})$ and $s=1$,

$$
\begin{aligned}
& M^{q(2-1 / p)}\left[P_{I}^{(1-1 / p)} \sum_{i \in I} p_{i} f\left(x_{i}\right)-P_{I}^{(2-1 / p)} f\left(\frac{1}{P_{I}} \sum_{i \in I} p_{i} x_{i}\right)\right]^{q} \\
& \geq\left[Q_{I}^{(1-1 / p)} \sum_{i \in I} q_{i} f\left(x_{i}\right)-Q_{I}^{(2-1 / p)} f\left(\frac{1}{Q_{I}} \sum_{i \in I} q_{i} x_{i}\right)\right]^{q} \\
& \geq m^{q(2-1 / p)}\left[P_{I}^{(1-1 / p)} \sum_{i \in I} p_{i} f\left(x_{i}\right)-P_{I}^{(2-1 / p)} f\left(\frac{1}{P_{I}} \sum_{i \in I} p_{i} x_{i}\right)\right]^{q} .
\end{aligned}
$$

Taking the power $1 / q>0$ in the inequality (3.5) we deduce the desired result (3.4).

The above Proposition 3.4 can be utilized to obtain various inequalities generated by the appropriate choices of the convex function $f$. 
(1) If $f: X \rightarrow \mathbb{R}, f(x)=\|x\|^{r}, r \geq 1, p>1$ where $(X,\|\cdot\|)$ is a normed linear space, then we can state the inequality

$$
\begin{aligned}
& M^{(2 p-1) / p}\left(\frac{P_{I}}{Q_{I}}\right)^{(p-1) / p}\left[\sum_{i \in I} p_{i}\left\|x_{i}\right\|^{r}-P_{I}^{1-r}\left\|\sum_{i \in I} p_{i} x_{i}\right\|^{r}\right] \\
& \geq \sum_{i \in I} q_{i}\left\|x_{i}\right\|^{r}-Q_{I}^{1-r}\left\|\sum_{i \in I} q_{i} x_{i}\right\|^{r} \\
& \geq m^{(2 p-1) / p}\left(\frac{P_{I}}{Q_{I}}\right)^{(p-1) / p}\left[\sum_{i \in I} p_{i}\left\|x_{i}\right\|^{r}-P_{I}^{1-r}\left\|\sum_{i \in I} p_{i} x_{i}\right\|^{r}\right]
\end{aligned}
$$

and, in particular,

$$
\begin{aligned}
& M^{(2 p-1) / p}\left(\frac{P_{I}}{Q_{I}}\right)^{(p-1) / p}\left[\sum_{i \in I} p_{i}\left\|x_{i}\right\|-\left\|\sum_{i \in I} p_{i} x_{i}\right\|\right] \\
& \geq \sum_{i \in I} q_{i}\left\|x_{i}\right\|-\left\|\sum_{i \in I} q_{i} x_{i}\right\| \\
& \geq m^{(2 p-1) / p}\left(\frac{P_{I}}{Q_{I}}\right)^{(p-1) / p}\left[\sum_{i \in I} p_{i}\left\|x_{i}\right\|-\left\|\sum_{i \in I} p_{i} x_{i}\right\|\right]
\end{aligned}
$$

for $I \in \mathcal{P}_{f}(\mathbb{N})$ and $\mathbf{p}, \mathbf{q} \in S_{+}(I)$ with $M \mathbf{p} \geq \mathbf{q} \geq m \mathbf{p}$ and $M \geq m>0$, for any vectors $x_{i} \in X, i \in I$.

(2) For $x_{i}>0$ and $p_{i} \geq 0(i \in \mathbb{N})$ so that $P_{I}>0$, let us denote

$$
A(I, \mathbf{p}, x):=\frac{1}{P_{I}} \sum_{i \in I} p_{i} x_{i}, \quad G(I, \mathbf{p}, x):=\left(\prod_{i \in I}\left(x_{i}\right)^{p_{i}}\right)^{1 / P_{I}},
$$

to be the weighted arithmetic and geometric means, respectively.

Applying the above Proposition 3.4 for the convex function $f(x)=-\ln x, x \in$ $(0, \infty)$, we can state the inequality

$$
\begin{aligned}
{\left[\frac{A(I, \mathbf{p}, x)}{G(I, \mathbf{p}, x)}\right]^{M^{(2 p-1) / p}\left(P_{I} / Q_{I}\right)^{(2 p-1) / p}} } & \geq \frac{A(I, \mathbf{q}, x)}{G(I, \mathbf{q}, x)} \\
& \geq\left[\frac{A(I, \mathbf{p}, x)}{G(I, \mathbf{p}, x)}\right]^{m^{(2 p-1) / p}\left(P_{I} / Q_{I}\right)^{(2 p-1) / p}}
\end{aligned}
$$

for $p>1, I \in \mathcal{P}_{f}(\mathbb{N})$ and $\mathbf{p}, \mathbf{q} \in S_{+}(I)$ with $M \mathbf{p} \geq \mathbf{q} \geq m \mathbf{p}$ and $M \geq m>0$, for any $x_{i}>0, i \in I$.

It is well known that if $f: C \rightarrow \mathbb{R}$ is a strictly convex function and not all vectors $x_{i} \in C, i \in I$ are equal between them, then a strict inequality holds in Jensen's result (3.1). Therefore, we can consider the functional

$$
R_{p, q, I}(\mathbf{p}):=\frac{1}{v^{q((1 / p)-1)}(x) h^{q}(x)}
$$


where, as above, $v(\mathbf{p})=P_{I}, h(\mathbf{p})=J_{I}(\mathbf{p})$ and $0<p, q<1$.

In a more explicit way we then have

$$
R_{p, q, I}(\mathbf{p})=\frac{1}{P_{I}^{q / p}\left[\frac{1}{P_{I}} \sum_{i \in I} p_{i} f\left(x_{i}\right)-f\left(\frac{1}{P_{I}} \sum_{i \in I} p_{i} x_{i}\right)\right]^{q}} .
$$

We can also consider the particular functional

$$
R_{p, I}(\mathbf{p})=\frac{1}{P_{I}\left[\frac{1}{P_{I}} \sum_{i \in I} p_{i} f\left(x_{i}\right)-f\left(\frac{1}{P_{I}} \sum_{i \in I} p_{i} x_{i}\right)\right]^{p}} .
$$

By utilizing Corollary 2.6, we can state the following result.

Proposition 3.5. If $f: C \rightarrow \mathbb{R}$ is a strictly convex function and not all vectors $x_{i} \in C, i \in I$ are equal between them, then the functional $R_{p, q, I}$ is subadditive on $S_{+}(I)$ for any $p, q$ with $0<p, q<1$.

As a simple example, we can consider the functional

$$
\lambda_{p, q, I}(\mathbf{p}):=\frac{1}{P_{I}^{q / p}\left[\ln \left(\frac{A(I, \mathbf{p}, x)}{G(I, \mathbf{p}, x)}\right)\right]^{p}},
$$

which is therefore subadditive on $S_{+}(I)$ for any $p, q$ with $0<p, q<1$ and any $x_{i}>0$, $i \in I$ that are not all equal between them.

\section{Applications for Hölder's and Minkowski's inequalities}

Let $(X,\|\cdot\|)$ be a normed space and $I \in \mathcal{P}_{f}(\mathbb{N})$. We define

$$
E(I):=\left\{x=\left(x_{j}\right)_{j \in I} \mid x_{j} \in X, j \in I\right\}
$$

and

$$
\mathbb{K}(I):=\left\{\lambda=\left(\lambda_{j}\right)_{j \in I} \mid \lambda_{j} \in \mathbb{K}, j \in I\right\} .
$$

We consider the functional

$$
H_{I}(\mathbf{p}, \lambda, x ; \alpha, \beta):=\left(\sum_{j \in I} p_{j}\left|\lambda_{j}\right|^{\alpha}\right)^{1 / \alpha}\left(\sum_{j \in I} p_{j}\left\|x_{j}\right\|^{\beta}\right)^{1 / \beta}-\left\|\sum_{j \in I} p_{j} \lambda_{j} x_{j}\right\| .
$$

for $\alpha, \beta>1$, where $1 / \alpha+1 / \beta=1$.

The following result has been proved in [3].

Lemma 4.1. For any $\mathbf{p}, \mathbf{q} \in S_{+}(I)$,

$$
H_{I}(\mathbf{p}+\mathbf{q}, \lambda, x ; \alpha, \beta) \geq H_{I}(\mathbf{p}, \lambda, x ; \alpha, \beta)+H_{I}(\mathbf{q}, \lambda, x ; \alpha, \beta),
$$

where $x \in E(I), \lambda \in \mathbb{K}(I)$ and $\alpha, \beta>1$ with $1 / \alpha+1 / \beta=1$. 
REMARK 4.2. The same result can be stated if $(B,\|\cdot\|)$ is a normed algebra and the functional $H$ is defined by

$$
H_{I}(\mathbf{p}, \lambda, x ; \alpha, \beta):=\left(\sum_{i \in I} p_{i}\left\|x_{i}\right\|^{\alpha}\right)^{1 / \alpha}\left(\sum_{i \in I} p_{i}\left\|y_{i}\right\|^{\beta}\right)^{1 / \beta}-\left\|\sum_{i \in I} p_{i} x_{i} y_{i}\right\|,
$$

where $x=\left(x_{i}\right)_{i \in I}, y=\left(y_{i}\right)_{i \in I} \subset B, \mathbf{p} \in S_{+}(I)$ and $\alpha, \beta>1$ with $1 / \alpha+1 / \beta=1$.

Define the following functional on $S_{+}(I)$ :

$$
H_{p, q, I}(\mathbf{p}, \lambda, x ; \alpha, \beta):=P_{I}^{q(1-(1 / p))} H_{I}^{q}(\mathbf{p}, \lambda, x ; \alpha, \beta) .
$$

Since $H(\cdot, \lambda, x ; \alpha, \beta)$ is positive homogeneous, on utilizing Corollary 2.4, we can state the following result.

Proposition 4.3. If $\mathbf{p}, \mathbf{q} \in S_{+}(I)$ and $M \geq m \geq 0$ with $M \mathbf{p} \geq \mathbf{q} \geq m \mathbf{p}$, then

$$
\begin{aligned}
& M^{(2 p-1) / p}\left(\frac{P_{I}}{Q_{I}}\right)^{(p-1) / p}\left[\left(\sum_{i \in I} p_{i}\left|\lambda_{i}\right|^{\alpha}\right)^{1 / \alpha}\left(\sum_{i \in I} p_{i}\left\|x_{i}\right\|^{\beta}\right)^{1 / \beta}-\left\|\sum_{i \in I} p_{i} \lambda_{i} x_{i}\right\|\right] \\
& \geq\left(\sum_{i \in I} q_{i}\left|\lambda_{i}\right|^{\alpha}\right)^{1 / \alpha}\left(\sum_{i \in I} q_{i}\left\|x_{i}\right\|^{\beta}\right)^{1 / \beta}-\left\|\sum_{i \in I} q_{i} \lambda_{i} x_{i}\right\| \\
& \geq m^{(2 p-1) / p}\left(\frac{P_{I}}{Q_{I}}\right)^{(p-1) / p}\left[\left(\sum_{i \in I} p_{i}\left|\lambda_{i}\right|^{\alpha}\right)^{1 / \alpha}\left(\sum_{i \in I} p_{i}\left\|x_{i}\right\|^{\beta}\right)^{1 / \beta}\right. \\
& \left.\quad-\left\|\sum_{i \in I} p_{i} \lambda_{i} x_{i}\right\|\right],
\end{aligned}
$$

for $x \in E(I), \lambda \in \mathbb{K}(I), p>1$ and $\alpha, \beta>1$, where $1 / \alpha+1 / \beta=1$.

PROOF. By Corollary 2.4 applied to the functional $\Psi_{p, q}(\mathbf{p})=H_{p, q, I}(\mathbf{p}, \lambda, x ; \alpha, \beta)$,

$$
\begin{aligned}
& M^{q(2-1 / p)} P_{I}^{q(1-1 / p)}\left[\left(\sum_{i \in I} p_{i}\left|\lambda_{i}\right|^{\alpha}\right)^{1 / \alpha}\left(\sum_{i \in I} p_{i}\left\|x_{i}\right\|^{\beta}\right)^{1 / \beta}-\left\|\sum_{i \in I} p_{i} \lambda_{i} x_{i}\right\|\right]^{q} \\
& \geq Q_{I}^{q(1-1 / p)}\left[\left(\sum_{i \in I} q_{i}\left|\lambda_{i}\right|^{\alpha}\right)^{1 / \alpha}\left(\sum_{i \in I} q_{i}\left\|x_{i}\right\|^{\beta}\right)^{1 / \beta}-\left\|\sum_{i \in I} q_{i} \lambda_{i} x_{i}\right\|\right]^{q} \\
& \geq m^{q(2-1 / p)} P_{I}^{q(1-1 / p)}\left[\left(\sum_{i \in I} p_{i}\left|\lambda_{i}\right|^{\alpha}\right)^{1 / \alpha}\left(\sum_{i \in I} p_{i}\left\|x_{i}\right\|^{\beta}\right)^{1 / \beta}\right. \\
& \left.\quad-\left\|\sum_{i \in I} p_{i} \lambda_{i} x_{i}\right\|\right]^{q} .
\end{aligned}
$$

Taking the power $1 / q>0$ in the inequality (4.4) we deduce the desired result (4.3). 
We define the functional

$$
\begin{aligned}
M_{I}(\mathbf{p}, x, y ; \alpha)=[ & \left.\left(\sum_{i \in I} p_{i}\left\|x_{i}\right\|^{\alpha}\right)^{1 / \alpha}+\left(\sum_{i \in I} p_{i}\left\|y_{i}\right\|^{\alpha}\right)^{1 / \alpha}\right]^{\alpha} \\
& -\sum_{i \in I} p_{i}\left\|x_{i}+y_{i}\right\|^{\alpha},
\end{aligned}
$$

where $\mathbf{p} \in S_{+}(I), \alpha \geq 1$ and $x, y \in E(I)$.

The following result concerning the superadditivity of the functional $M_{I}(\cdot, x, y ; \alpha)$ holds [3].

LEMMA 4.4. For any $\mathbf{p}, \mathbf{q} \in S_{+}(I)$,

$$
M_{I}(\mathbf{p}+\mathbf{q}, x, y ; \alpha) \geq M_{I}(\mathbf{p}, x, y ; \alpha)+M_{I}(\mathbf{q}, x, y ; \alpha),
$$

where $x, y \in E(I)$ and $\alpha \geq 1$.

Since the functional $M_{I}(\cdot, x, y ; \alpha)$ is positive homogeneous on $S_{+}(I)$, then on utilizing Corollary 2.4, we can state the following proposition.

Proposition 4.5. If $\mathbf{p}, \mathbf{q} \in S_{+}(I)$ and $M \geq m \geq 0$ with $M \mathbf{p} \geq \mathbf{q} \geq m \mathbf{p}$, then

$$
\begin{aligned}
& M^{(2 p-1) / p}\left(\frac{P_{I}}{Q_{I}}\right)^{(p-1) / p}\left\{\left[\left(\sum_{i \in I} p_{i}\left\|x_{i}\right\|^{\alpha}\right)^{1 / \alpha}+\left(\sum_{i \in I} p_{i}\left\|y_{i}\right\|^{\alpha}\right)^{1 / \alpha}\right]^{\alpha}\right. \\
& \left.-\sum_{i \in I} p_{i}\left\|x_{i}+y_{i}\right\|^{\alpha}\right\} \\
& \geq\left[\left(\sum_{i \in I} q_{i}\left\|x_{i}\right\|^{\alpha}\right)^{1 / \alpha}+\left(\sum_{i \in I} q_{i}\left\|y_{i}\right\|^{\alpha}\right)^{1 / \alpha}\right]^{\alpha}-\sum_{i \in I} q_{i}\left\|x_{i}+y_{i}\right\|^{\alpha} \\
& \geq m^{(2 p-1) / p}\left(\frac{P_{I}}{Q_{I}}\right)^{(p-1) / p}\left\{\left[\left(\sum_{i \in I} p_{i}\left\|x_{i}\right\|^{\alpha}\right)^{1 / \alpha}+\left(\sum_{i \in I} p_{i}\left\|y_{i}\right\|^{\alpha}\right)^{1 / \alpha}\right]^{\alpha}\right. \\
& \left.\quad-\sum_{i \in I} p_{i}\left\|x_{i}+y_{i}\right\|^{\alpha}\right\},
\end{aligned}
$$

where $x, y \in E(I), p>1$ and $\alpha \geq 1$.

\section{Applications for Schwarz's inequality}

Let $X$ be a linear space over the real or complex number field $\mathbb{K}$ and let us denote by $\mathcal{H}(X)$ the class of all positive semi-definite Hermitian forms on $X$ or, for simplicity, nonnegative forms on $X$, that is, the mapping $\langle\cdot, \cdot\rangle: X \times X \rightarrow \mathbb{K}$ belongs to $\mathcal{H}(X)$ if it satisfies the conditions:

(i) $\langle x, x\rangle \geq 0$ for all $x \in X$;

(ii) $\langle\alpha x+\beta y, z\rangle=\alpha\langle x, z\rangle+\beta\langle y, z\rangle$ for all $x, y \in X$ and $\alpha, \beta \in \mathbb{K}$;

(iii) $\langle y, x\rangle=\overline{\langle x, y\rangle}$ for all $x, y \in X$. 
If $\langle\cdot, \cdot\rangle \in \mathcal{H}(X)$, then the functional $\|\cdot\|=\langle\cdot, \cdot\rangle^{1 / 2}$ is a semi-norm on $X$ and the following version of Schwarz's inequality holds:

$$
\|x\|\|y\| \geq|\langle x, y\rangle|
$$

for each $x, y \in H$.

Now, let us observe that $\mathcal{H}(X)$ is a convex cone in the linear space of all mappings defined on $X^{2}$ with values in $\mathbb{K}$. Also, we can introduce on $\mathcal{H}(X)$ the following binary relation $[4]$ :

$$
\langle\cdot, \cdot\rangle_{2} \geq\langle\cdot, \cdot\rangle_{1} \text { if and only if }\|x\|_{2} \geq\|x\|_{1} \text { for any } x \in H .
$$

This is an order relation on $\mathcal{H}(X)$ (see [4]).

Consider the functional [4]

$$
\sigma: \mathcal{H}(X) \times X^{2} \rightarrow \mathbb{R}_{+}, \quad \sigma(\langle\cdot, \cdot\rangle ; x, y):=\|x\|\|y\|-|\langle x, y\rangle|,
$$

which is closely related to the Schwarz inequality in (5.1).

LEMMA 5.1 [4]. The functional $\sigma(\cdot ; x, y)$ is nonnegative, superadditive and positive homogeneous on $\mathcal{H}(X)$.

Now, if we consider the composite functional

$$
\begin{aligned}
\Psi_{p, q, e}(\langle\cdot, \cdot\rangle, x, y) & =\langle e, e\rangle^{q(1-(1 / p))} \sigma^{q}(\langle\cdot, \cdot\rangle ; x, y) \\
& =\|e\|^{2 q(1-(1 / p))}[\|x\|\|y\|-|\langle x, y\rangle|]^{q}
\end{aligned}
$$

where $x, y, z \in X$, and $p, q \geq 1$, then, by Corollary 2.2, we can state the following result.

PROPOSITION 5.2. The functional $\Psi_{p, q, e}(\cdot, x, y)$ is superadditive and monotonic nondecreasing on $\mathcal{H}(X)$.

The following proposition concerning some inequalities for equivalent norms generated by inner products is of interest for applications.

Proposition 5.3. Let $M \geq m>0$ and let $\langle\cdot, \cdot\rangle_{1},\langle\cdot, \cdot\rangle_{2}$ be two inner products on $X$ such that $M\|x\|_{1} \geq\|x\|_{2} \geq m\|x\|_{1}$ for each $x \in X$. If $e \in X, e \neq 0$ and $p \geq 1$, then

$$
\begin{gathered}
M^{2(2 p-1) / p}\left(\frac{\|e\|_{1}}{\|e\|_{2}}\right)^{2(p-1) / p}\left(\|x\|_{1}\|y\|_{1}-\left|\langle x, y\rangle_{1}\right|\right) \geq\|x\|_{2}\|y\|_{2}-\left|\langle x, y\rangle_{2}\right| \\
\geq m_{1}^{2(2 p-1) / p}\left(\frac{\|e\|_{1}}{\|e\|_{2}}\right)^{2(p-1) / p}\left(\|x\|_{1}\|y\|_{1}-\left|\langle x, y\rangle_{1}\right|\right),
\end{gathered}
$$

for any $x, y \in H$. 
PROOF. On applying Corollary 2.4, we deduce that

$$
\begin{aligned}
& M^{2 q(2-(1 / p))}\|e\|_{1}^{2 q(1-1 / p)}\left[\|x\|_{1}\|y\|_{1}-\left|\langle x, y\rangle_{1}\right|\right]^{q} \\
& \quad \geq\|e\|_{2}^{2 q(1-1 / p)}\left[\|x\|_{2}\|y\|_{2}-\left|\langle x, y\rangle_{2}\right|\right]^{q} \\
& \quad \geq m^{2 q(2-1 / p)}\|e\|_{1}^{2 q(1-1 / p)}\left[\|x\|_{1}\|y\|_{1}-\left|\langle x, y\rangle_{1}\right|\right]^{q}
\end{aligned}
$$

for $x, y$ fixed in $X$.

Taking the power $1 / q>0$ in the inequality (5.5), we deduce the desired result (5.4).

The above result can be used to obtain some inequalities for positive definite operators.

Corollary 5.4. Assume that $A: H \rightarrow H$ is a self-adjoint linear operator on the Hilbert space $(H,\langle\cdot, \cdot\rangle)$ satisfying the property that there exist $\Gamma \geq \gamma>0$ such that $\Gamma I \geq A \geq \gamma I$ in the operator order (that is, $\Gamma\|x\|^{2} \geq\langle A x, x\rangle \geq \gamma\|x\|^{2}$ for any $x \in H$ ). Then for $p \geq 1$ we have the inequality

$$
\begin{aligned}
& \frac{\Gamma^{(2 p-1) / p}}{\langle A e, e\rangle^{(p-1) / p}}(\|x\|\|y\|-|\langle x, y\rangle|) \geq\langle A x, x\rangle^{1 / 2}\langle A y, y\rangle^{1 / 2}-|\langle A x, y\rangle| \\
& \geq \frac{\gamma^{(2 p-1) / p}}{\langle A e, e\rangle^{(p-1) / p}}(\|x\|\|y\|-|\langle x, y\rangle|),
\end{aligned}
$$

for any $x, y \in H$ and $e \in H$ with $\|e\|=1$.

The following result for invertible operators also holds.

Corollary 5.5. Assume that $A: H \rightarrow H$ is an invertible linear operator on the Hilbert space $(H,\langle\cdot, \cdot\rangle)$. Then for $p \geq 1$ and $e \in H$ with $\|e\|=1$ we have the inequality

$$
\begin{gathered}
\frac{\|A\|^{2((2 p-1) / p)}}{\|A e\|^{2((p-1) / p)}}(\|x\|\|y\|-|\langle x, y\rangle|) \geq\|A x\|\|A y\|-|\langle A x, A y\rangle| \\
\geq \frac{\left\|A^{-1}\right\|^{-2((2 p-1) / p)}}{\|A e\|^{2((p-1) / p)}}(\|x\|\|y\|-|\langle x, y\rangle|),
\end{gathered}
$$

for any $x, y \in H$.

Proof. Since $A$ is invertible,

$$
\|A\|\|x\| \geq\|A x\| \geq\left\|A^{-1}\right\|^{-1}\|x\|
$$

for any $x \in H$. Applying Proposition 5.3 we deduce the desired result (5.7). 
REMARK 5.6. Similar results can be stated if one uses the following nonnegative, superadditive and positive homogeneous functionals on $\mathcal{H}(X)$ (see [2, pp. 8-15]):

$$
\begin{gathered}
\sigma_{r}(\langle\cdot, \cdot\rangle ; x, y):=\|x\|\|y\|-\operatorname{Re}\langle x, y\rangle, \\
\delta(\langle\cdot, \cdot\rangle ; x, y):=\|x\|^{2}\|y\|^{2}-|\langle x, y\rangle|^{2}, \\
\delta_{r}(\langle\cdot, \cdot\rangle ; x, y):=\|x\|^{2}\|y\|^{2}-(\operatorname{Re}\langle x, y\rangle)^{2}, \\
\gamma(\langle\cdot, \cdot\rangle ; x, y):=\frac{\|x\|^{2}\|y\|^{2}-|\langle x, y\rangle|^{2}}{\|y\|^{2}},
\end{gathered}
$$

where, in the definition of $\gamma,\langle\cdot, \cdot\rangle$ is an inner product and $y$ is not zero, and

$$
\beta(\langle\cdot, \cdot\rangle ; x, y):=\left(\|x\|^{2}\|y\|^{2}-|\langle x, y\rangle|^{2}\right)^{\frac{1}{2}},
$$

for each $x, y \in X$.

The details are left to the interested reader.

REMARK 5.7. For other examples of superadditive (subadditive) functionals that can provide interesting inequalities similar to the ones outlined above, we refer to [1, 6-9].

\section{References}

[1] H. Alzer, 'Sub- and superadditive properties of Euler's gamma function', Proc. Amer. Math. Soc. 135(11) (2007), 3641-3648.

[2] S. S. Dragomir, Advances in Inequalities of the Schwarz, Triangle and Heisenberg Type in Inner Product Spaces (Nova Science Publishers, New York, 2007).

[3] S. S. Dragomir, 'Inequalities for superadditive functionals with applications', Bull. Aust. Math. Soc. 77 (2008), 401-411.

[4] S. S. Dragomir and B. Mond, 'On the superadditivity and monotonicity of Schwarz's inequality in inner product spaces', Contributions, Macedonian Acad. Sci. Arts 15(2) (1994), 5-22.

[5] S. S. Dragomir, J. Pečarić and L. E. Persson, 'Properties of some functionals related to Jensen's inequality', Acta Math. Hungar. 70 (1996), 129-143.

[6] L. Losonczi, 'Sub- and superadditive integral means', J. Math. Anal. Appl. 307(2) (2005), 444-454.

[7] S. Y. Trimble, J. Wells and F. T. Wright, 'Superadditive functions and a statistical application', SIAM J. Math. Anal. 20(5) (1989), 1255-1259.

[8] A. Vitolo and U. Zannier, 'Inequalities for superadditive functions related to a property R [of] convex sets', Boll. Unione Mat. Ital. A (7) 4(3) (1990), 309-314.

[9] P. Volkmann, 'Sur les fonctions simultanément suradditives et surmultiplicatives', Bull. Math. Soc. Sci. Math. R. S. Roumanie (N.S.) 28(76)(2) (1984), 181-184.

\section{S. S. DRAGOMIR, Mathematics, School of Engineering \& Science,} Victoria University, PO Box 14428, Melbourne City, MC 8001, Australia and

School of Computational and Applied Mathematics, University of the Witwatersrand, Private Bag-3, Wits-2050, Johannesburg, South Africa

e-mail: sever.dragomir@vu.edu.au 